\title{
Complete Genome Sequence of Staphylococcus aureus Siphophage Lorac
}

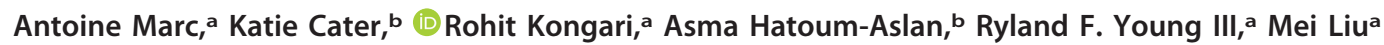 \\ aCenter for Phage Technology, Texas A\&M University, College Station, Texas, USA \\ bDepartment of Biological Sciences, The University of Alabama, Tuscaloosa, Alabama, USA
}

ABSTRACT Staphylococcus aureus is a leading cause of a wide range of clinical infections. Here, we announce the complete genome sequence of $S$. aureus siphophage Lorac, a phiETA-like temperate phage that is similar at the nucleotide level to the previously described $S$. aureus prophage phiNM2.

taphylococcus aureus is a Gram-positive skin-associated bacterium and is the causative agent for a wide range of clinical infections, including bacteremia, infective endocarditis, and pleuropulmonary and other device-related infections (1). Temperate phages, such as phiETA, are able to move between $S$. aureus strains and can encode virulence factors or toxins (2).

Siphophage Lorac was isolated from the wastewater treatment plant in Tuscaloosa, Alabama, in August 2015 using the host strain S. aureus strain RN4220. Host bacteria were cultured on tryptic soy broth or agar (Difco) at $37^{\circ} \mathrm{C}$ with aeration. Phages were cultured and propagated by the soft agar overlay method (3). It was identified as a siphophage using negative-stain transmission electron microscopy performed at the University of Alabama Optical Analysis Facility, as described previously (4). Phage genomic DNA was prepared using a modified Promega Wizard DNA cleanup kit protocol as described previously (4). Pooled indexed DNA libraries were prepared using the Illumina TruSeq Nano low-throughput (LT) kit, and the sequence was obtained from the Illumina MiSeq platform using the MiSeq v2 500-cycle reagent kit following the manufacturer's instructions, producing 631,646 reads for the index containing the phage genome. FastQC 0.11 .5 (https://www.bioinformatics.babraham.ac.uk/projects/ fastqc/) was used to quality control reads. The reads were trimmed with FastX-Toolkit 0.0.14 (http://hannonlab.cshl.edu/fastx_toolkit/download.html) before being assembled using SPAdes 3.5 .0 (5). Contig completion was confirmed by PCR using primers (5'-GTCCCTATCAAACCGAGAATCC-3' and 5'-ACATGGGTGTAATCGACAAAGA-3') facing off the ends of the assembled contig and Sanger sequencing of the resulting product, with the contig sequence manually corrected to match the resulting Sanger sequencing read. GLIMMER 3.0 (6) and MetaGeneAnnotator 1.0 (7) were used to predict protein-coding genes with manual correction for appropriate gene starts, and tRNA genes were predicted with ARAGORN 2.36 (8). Rho-independent termination sites were identified via TransTerm (http://transterm.cbcb.umd.edu/). Sequence similarity searches were performed by BLASTp 2.2.28 (9) against the NCBI nonredundant (nr), UniProt Swiss-Prot (10), and TrEMBL databases. InterProScan 5.15-54.0 (11), LipoP (12), and TMHMM v2.0 (13) were used to predict protein function. All analyses were conducted at default settings via the CPT Galaxy (14) and WebApollo (15) interfaces (https://cpt .tamu.edu/galaxy-pub).

Lorac was assembled as a complete genome with 43,147 bp and 1,371-fold coverage. It has a G+C content of $34 \%$, which is similar to that of its host (16). Lorac is probably a temperate phage since it belongs to the Phietavirus genus and is $99.18 \%$
Citation Marc A, Cater K, Kongari R, HatoumAslan A, Young RF, III, Liu M. 2019. Complete genome sequence of Staphylococcus aureus siphophage Lorac. Microbiol Resour Announc 8:e00586-19. https://doi.org/10.1128/MRA .00586-19.

Editor John J. Dennehy, Queens College Copyright $\odot 2019$ Marc et al. This is an openaccess article distributed under the terms of the Creative Commons Attribution 4.0 International license.

Address correspondence to Asma HatoumAslan, asma.hatoum@ua.edu, or Mei Liu, meiliu@tamu.edu.

Received 17 May 2019

Accepted 30 May 2019

Published 3 July 2019 
similar at the nucleotide level to phiNM2 (GenBank accession no. DQ530360), a prophage identified in S. aureus strain Newman (17). Morphogenesis genes coding for the major capsid protein, scaffolding protein, TerL, TerS, tail proteins, tail-head connector protein, portal protein, tape measure protein, and tape measure chaperone were annotated. Lysogeny-associated proteins, including Cro-like and cl-like regulators, excisionase, and integrase, were identified, as was a lysis cassette consisting of a class II holin and an amidase-type endolysin.

Data availability. The genome sequence of phage Lorac was submitted to GenBank under accession no. MH321492. Associated BioProject, SRA, and BioSample accession numbers are PRJNA222858, SRR8788599, and SAMN11260821, respectively.

\section{ACKNOWLEDGMENTS}

This work was supported by funding from the National Science Foundation (awards EF-0949351, DBI-1565146, and MCB-1749886). Additional support came from the Center for Phage Technology (CPT), an Initial University Multidisciplinary Research Initiative supported by Texas A\&M University and Texas AgriLife, and from the Department of Biochemistry and Biophysics at Texas A\&M University.

We are grateful for the advice and support of the CPT staff and Kim Lackey at the University of Alabama Optical Analysis Facility.

This phage was discovered in a pilot run for BSC 411 Phage Discovery, an undergraduate course at the University of Alabama. This announcement was prepared in partial fulfillment of the requirements for $\mathrm{BICH} 464$ Phage Genomics, an undergraduate course at Texas A\&M University.

\section{REFERENCES}

1. Tong SYC, Davis JS, Eichenberger E, Holland TL, Fowler VG, Jr. 2015. Staphylococcus aureus infections: epidemiology, pathophysiology, clinical manifestations, and management. Clin Microbiol Rev 28:603-661. https://doi.org/10.1128/CMR.00134-14.

2. Yamaguchi T, Hayashi T, Takami H, Nakasone K, Ohnishi M, Nakayama K, Yamada S, Komatsuzawa H, Sugai M. 2000. Phage conversion of exfoliative toxin A production in Staphylococcus aureus. Mol Microbiol 38: 694-705. https://doi.org/10.1046/j.1365-2958.2000.02169.x.

3. Adams MK. 1959. Bacteriophages. Interscience Publishersm Inc., New York, NY.

4. Cater K, Dandu VS, Bari SM, Lackey K, Everett GF, Hatoum-Aslan A. 2017. A novel Staphylococcus podophage encodes a unique lysin with unusual modular design. mSphere 2:e00040-17. https://doi.org/10.1128/ mSphere.00040-17.

5. Bankevich A, Nurk S, Antipov D, Gurevich AA, Dvorkin M, Kulikov AS, Lesin VM, Nikolenko SI, Pham S, Prjibelski AD, Pyshkin AV, Sirotkin AV, Vyahhi N, Tesler G, Alekseyev MA, Pevzner PA. 2012. SPAdes: a new genome assembly algorithm and its applications to single-cell sequencing. J Comput Biol 19:455-477. https://doi.org/10.1089/cmb.2012.0021.

6. Delcher AL, Harmon D, Kasif S, White O, Salzberg SL. 1999. Improved microbial gene identification with GLIMMER. Nucleic Acids Res 27: 4636-4641. https://doi.org/10.1093/nar/27.23.4636.

7. Noguchi H, Taniguchi T, Itoh T. 2008. MetaGeneAnnotator: detecting species-specific patterns of ribosomal binding site for precise gene prediction in anonymous prokaryotic and phage genomes. DNA Res 15:387-396. https://doi.org/10.1093/dnares/dsn027.

8. Laslett D, Canback B. 2004. ARAGORN, a program to detect tRNA genes and tmRNA genes in nucleotide sequences. Nucleic Acids Res 32:11-16. https://doi.org/10.1093/nar/gkh152.

9. Camacho C, Coulouris G, Avagyan V, Ma N, Papadopoulos J, Bealer K,
Madden TL. 2009. BLAST+: architecture and applications. BMC Bioinformatics 10:421. https://doi.org/10.1186/1471-2105-10-421.

10. The UniProt Consortium. 2018. UniProt: the universal protein knowledgebase. Nucleic Acids Res 46:2699. https://doi.org/10.1093/nar/ gky092.

11. Jones $P$, Binns $D$, Chang $H Y$, Fraser $M$, Li W, McAnulla $C, M c$ William $H_{\text {, }}$ Maslen J, Mitchell A, Nuka G, Pesseat S, Quinn AF, Sangrador-Vegas A, Scheremetjew M, Yong SY, Lopez R, Hunter S. 2014. InterProScan 5: genome-scale protein function classification. Bioinformatics 30:1236-1240. https://doi.org/10.1093/bioinformatics/btu031.

12. Juncker AS, Willenbrock $H$, Von Heijne $G$, Brunak $S$, Nielsen $H$, Krogh $A$. 2003. Prediction of lipoprotein signal peptides in Gram-negative bacteria. Protein Sci 12:1652-1662. https://doi.org/10.1110/ps.0303703.

13. Krogh A, Larsson B, von Heijne G, Sonnhammer EL. 2001. Predicting transmembrane protein topology with a hidden Markov model: application to complete genomes. J Mol Biol 305:567-580. https://doi.org/10 .1006/jmbi.2000.4315

14. Cock PJ, Gruning BA, Paszkiewicz K, Pritchard L. 2013. Galaxy tools and workflows for sequence analysis with applications in molecular plant pathology. PeerJ 1:e167. https://doi.org/10.7717/peerj.167.

15. Lee E, Helt GA, Reese JT, Munoz-Torres MC, Childers CP, Buels RM, Stein L, Holmes IH, Elsik CG, Lewis SE. 2013. Web Apollo: a Web-based genomic annotation editing platform. Genome Biol 14:R93. https://doi .org/10.1186/gb-2013-14-8-r93.

16. Suzuki H, Lefébure T, Bitar PP, Stanhope MJ. 2012. Comparative genomic analysis of the genus Staphylococcus including Staphylococcus aureus and its newly described sister species Staphylococcus simiae. BMC Genomics 13:38. https://doi.org/10.1186/1471-2164-13-38.

17. Bae T, Baba T, Hiramatsu K, Schneewind O. 2006. Prophages of Staphylococcus aureus Newman and their contribution to virulence. Mol Microbiol 62:1035-1047. https://doi.org/10.1111/j.1365-2958.2006.05441.x. 